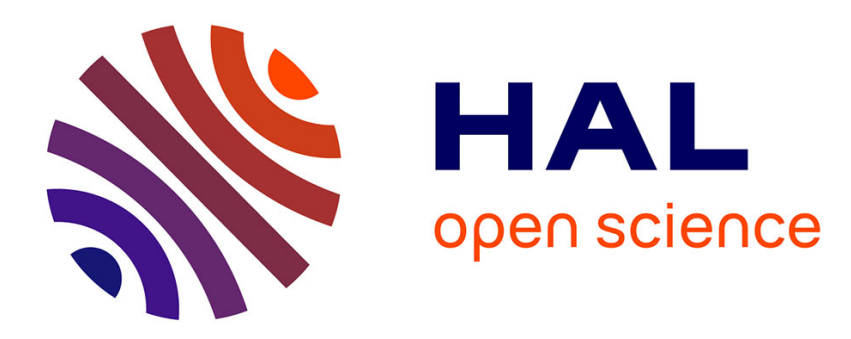

\title{
Winning coalitions in plurality voting democracies
}

René van den Brink, Dinko Dimitrov, Agnieszka Rusinowska

\section{To cite this version:}

René van den Brink, Dinko Dimitrov, Agnieszka Rusinowska. Winning coalitions in plurality voting democracies. Social Choice and Welfare, 2021, 56, pp.509-530. 10.1007/s00355-020-01290-y . hal03153465

\section{HAL Id: hal-03153465 \\ https://hal.science/hal-03153465}

Submitted on 31 Aug 2021

HAL is a multi-disciplinary open access archive for the deposit and dissemination of scientific research documents, whether they are published or not. The documents may come from teaching and research institutions in France or abroad, or from public or private research centers.
L'archive ouverte pluridisciplinaire HAL, est destinée au dépôt et à la diffusion de documents scientifiques de niveau recherche, publiés ou non, émanant des établissements d'enseignement et de recherche français ou étrangers, des laboratoires publics ou privés.

\section{(c)(1)}

Distributed under a Creative Commons Attribution| 4.0 International License 


\title{
Winning coalitions in plurality voting democracies
}

\author{
René van den Brink ${ }^{1}$ Dinko Dimitrov² ${ }^{\mathbb{D}} \cdot$ Agnieszka Rusinowska $^{3}$
}

Received: 3 November 2019 / Accepted: 19 September 2020 / Published online: 6 October 2020

(c) The Author(s) 2020

\begin{abstract}
We consider plurality voting games being simple games in partition function form such that in every partition there is at least one winning coalition. Such a game is said to be weighted if it is possible to assign weights to the players in such a way that a winning coalition in a partition is always one for which the sum of the weights of its members is maximal over all coalitions in the partition. A plurality game is called decisive if in every partition there is exactly one winning coalition. We show that in general, plurality games need not be weighted, even not when they are decisive. After that, we prove that (i) decisive plurality games with at most four players, (ii) majority games with an arbitrary number of players, and (iii) decisive plurality games that exhibit some kind of symmetry, are weighted. Complete characterizations of the winning coalitions in the corresponding partitions are provided as well.
\end{abstract}

\section{Introduction}

In parliamentary voting, a plurality voting system is a system where each voter can vote for one party, and the seats in parliament are allocated to the parties dependent on (usually proportional to) the cast votes. If one party received the majority of

\footnotetext{
We are grateful to Francis Bloch and Anirban Kar for fruitful discussions, as well as to four anonymous referees for their helpful comments and suggestions.

Dinko Dimitrov

dinko.dimitrov@mx.uni-saarland.de

René van den Brink

jrbrink@feweb.vu.nl

Agnieszka Rusinowska

agnieszka.rusinowska@univ-paris1.fr

1 Department of Econometrics and OR, and Tinbergen Institute, VU University, De Boelelaan 1105, 1081 HV Amsterdam, The Netherlands

2 Chair of Economic Theory, Saarland University, Campus C3 1, 66123 Saarbrücken, Germany

3 CNRS-Paris School of Economics, Centre d'Economie de la Sorbonne, 106-112 Bd de l'Hôpital, 75647 Paris Cedex 13, France
} 
the votes, then it can be considered the winner of the election. However, very often there is no majority party, and two or more parties need to cooperate and make an agreement to form a (majority) government. Although in such situations there is no party that can guarantee for itself to be in the government, and thus it is not immediately clear who is the winner of the election, usually immediately after the elections there is at least one party that claims to be the winner of the election. For instance, the party with the most votes/seats often declares itself as the winner of the election. But if other, smaller, parties form a majority coalition, then the party with the most votes might eventually be the 'loser'. For example, if after election a right wing party received the most votes, but the left wing parties can form a majority coalition, then it might be that the largest, or most moderate, left wing party can be considered to be the winner of the election.

Typically, in such situations, whether a coalition is winning or losing might depend on the way how players outside the coalition are organized into coalitions. Therefore, in this paper, we frame debates about winners of an election in the setup of simple cooperative games in partition function form. We address the question if it is possible to assign weights to political parties that somehow measure who is the winner of an election. If that is possible, we say that the corresponding simple game supports a plurality voting democracy.

We introduce plurality games as a special type of simple games in partition function form. A classical simple game assigns to every coalition (i.e., subset of the player set) either a worth of one (if the coalition is winning) or zero (if the coalition is losing). In such a game, whether a coalition is winning or losing does not depend on the way how the players/parties outside the coalition are organized. This assumption is reasonable when we define a coalition to be winning if it has a majority, since in that case any coalition of the opposing parties is a minority coalition. However, in this paper we want to address the issue of determining the winner or strongest party in a coalitional configuration of parties, even when there is no majority coalition formed (yet). During the process of government formation, different potential compositions of government parties might be considered, and the parties trying to form a government use coalition formation with parties outside the current government negotiations in strengthening their arguments or bargaining positions.

Usually, in these cases, the strength of parties also depends on the way other parties form alliances, i.e., there are externalities of coalition formation in the sense that parties forming a coalition has impact on the strength of parties outside the coalition. Such situations can be modeled by simple games in partition function form, where a worth is assigned to every so-called embedded coalition being a pair consisting of a coalition and a partition that contains this coalition. The worth is one (respectively zero) if the corresponding coalition is winning (respectively losing) in the partition. We call such a game a plurality game, if in every partition there is at least one coalition that wins in the partition. So, winning does not necessarily mean that the coalition has a majority and can pass a bill, but simply that it is considered as the strongest in a given coalitional configuration as represented by the partition. For example, it is common practice that the party that got the most votes in an election is considered as a winner and takes the initiative to form a government. Although this does not imply that eventually this party will be in the government, it 
obviously gives the party an advantage as long as no coalitions are formed yet. On the other hand, it might be that a party that is not the largest, but has ideologically close parties that are big enough to form a majority coalition with, will eventually form a government, and thus can also be considered a winner after the elections. In that case there can be two (or even more) parties that are declared to be winner of the election.

In this paper, we assume plurality games to be monotonic, both with respect to a coalition as well as to a certain type of externalities regarding other coalitions. Specifically, we assume that (i) a winning coalition cannot become losing when it grows, and (ii) there are negative externalities of other coalitions growing in the sense that bigger outside coalitions give 'more resistance' and thus outside coalitions becoming bigger cannot turn a losing coalition into a winning one. ${ }^{1}$

Within this model of (monotonic) plurality games, we study the possibility of assigning weights to players (parties) such that a winning coalition in a partition is always one that has the maximal sum of its players' weights over all coalitions in the partition. If this is possible for a given game, then we call the game weighted and refer to the corresponding weights as supporting weights. In that case, we can say that the game supports a plurality voting democracy. In the following, we sometimes shortly speak about the weight of a coalition as being the sum of the weights of the players in the coalition. Notice that a game being weighted does not imply that, when two coalitions in a partition have maximal weight, then both are winning. It only requires that the winner should be one of the coalitions with maximal weight.

In this paper, an important role is played by decisive plurality games being plurality games, where each partition contains exactly one winning coalition. In Sect. 2, we illustrate the problem of assigning supporting weights to plurality games by providing an example of a five-player decisive plurality game which is not weighted. We start our investigation of finding plurality games that are weighted in Sect. 3, by showing that all games with at most four players are weighted. In Sect. 4, we show that a majority game (i.e., a plurality game such that the winner in every partition is a coalition of maximal size in the partition) with more than four players is weighted. We also define a notion of symmetry, and show that such symmetric majority games can only be supported by assigning equal weights to all players. Notice that, intuitively, not all players can be symmetric in a decisive plurality game, because for such a game exactly one singleton is winning in the partition that consists of all singletons. We refer to this special partition into singletons as the atomistic partition. It describes, for example, the situation directly after elections when no coalitions or alliances are formed yet.

The closest we can get to symmetry in a decisive plurality game is to require that all players but one (the winner in the atomistic partition) are symmetric. We call such games $(n-1)$-symmetric and show in Sect. 5 that $(n-1)$-symmetric decisive plurality games with an arbitrary number of players are weighted. For this, we

\footnotetext{
${ }^{1}$ In this paper, we focus on negative externalities for the reason explained in the text, but notice that there can also be situations of positive externalities, where coalition formation of other parties strengthens the position of a party. Such situations are not considered here.
} 
define the power of the winner in the atomistic partition in a specific way, show how it shapes the structure of the possible candidates for a winning coalition in a partition, and explicitly use it in the construction of supporting weights.

Section 6 concludes the main part of the paper and provides an overview of the related literature. All proofs are collected in Appendix A (proofs from Sect. 3), Appendix B (proofs from Sect. 4), and Appendix C (proofs from Sect. 5).

\section{Plurality games}

All games we consider will be defined on a fixed and finite player set $N=\{1, \ldots, n\}$ with $n \geq 2$, whose non-empty subsets are called coalitions. A collection $\pi$ of coalitions is called a coalition structure if $\pi$ is a partition of $N$, i.e., if all coalitions in $\pi$ are non-empty, pair-wise disjoint, and their union is $N$. We denote by $\mathcal{P}$ the set of all partitions (coalition structures) of $N$. For $\pi \in \mathcal{P}$ and $i \in N$, the notation $\pi(i)$ stands for the coalition in $\pi$ containing player $i$. The partition $\pi^{a} \in \mathcal{P}$ with $\pi^{a}(i)=\{i\}$ for each $i \in N$, is called the atomistic partition. A pair $(S ; \pi)$ consisting of a non-empty coalition $S \subseteq N$ and a partition $\pi \in \mathcal{P}$ with $S \in \pi$ is called an embedded coalition. The set of all embedded coalitions is $E=\left\{(S ; \pi) \in\left(2^{N} \backslash\{\emptyset\}\right) \times \mathcal{P} \mid S \in \pi\right\}$.

For partition $\pi \in \mathcal{P}$ and set of players $S \subset N$, we denote by $\pi_{S}=\{T \cap S \mid T \in \pi: T \cap S \neq \emptyset\}$ the partition of $S$ induced by $\pi$. Further, we will often write $\left\{T_{1}, \ldots, T_{k}, \pi_{S}\right\}$ for $\left\{T_{1}, \ldots, T_{k}, S_{1}, \ldots, S_{p}\right\}$ if $\pi_{S}=\left\{S_{1}, \ldots, S_{p}\right\}$.

Simple games in partition function form A simple game in partition function form is a pair $(N, v)$, where the partition function $v: E \rightarrow\{0,1\}$ is such that $v(N ;\{N\})=1$. An embedded coalition $(S ; \pi) \in E$ is winning in the game $(N, v)$ if and only if $v(S ; \pi)=1$. Otherwise, it is called losing. We sometimes say that coalition $S$ is winning in partition $\pi$ when $(S ; \pi)$ is a winning embedded coalition. The set of all winning embedded coalitions in the game $v$ is denoted by $E_{W}(v)$. Notice that this game form allows to model externalities of coalition formation. For instance, it can be that a coalition contained in two partitions $\pi$ and $\pi^{\prime}$ is winning in $\pi$ but losing in $\pi^{\prime}$. Since the player set $N$ is fixed, we often write a simple game in partition function form $(N, v)$ by its partition function $v$. We use the following notion of inclusion, borrowed from Alonso-Meijide et al. (2017): For $\left(S^{\prime} ; \pi^{\prime}\right),(S ; \pi) \in E$, we say that $\left(S^{\prime} ; \pi^{\prime}\right)$ is weakly included in $(S ; \pi)$, denoted by $\left(S^{\prime} ; \pi^{\prime}\right) \subseteq(S ; \pi)$, if (i) $S^{\prime} \subseteq S$, and (ii) for each $T \in \pi \backslash\{S\}$, there exists $T^{\prime} \in \pi^{\prime}$ with $T \subseteq T^{\prime}$. A game $v$ is then defined as monotonic if $\left(S^{\prime} ; \pi^{\prime}\right),(S ; \pi) \in E$ with $\left(S^{\prime} ; \pi^{\prime}\right) \subseteq(S ; \pi)$ implies $v\left(S^{\prime} ; \pi^{\prime}\right) \leq v(S ; \pi)$. This monotonicity notion reflects (i) a nonnegative effect when a coalition grows, and (ii) an idea of negative externalities when players outside a coalition form larger coalitions. In particular, it implies that when a coalition is winning in a partition, then it is winning in every finer partition that contains this coalition. In other words, the idea expressed here is that in a finer partition there is 'less resistance' against the winning coalition. Clearly, a winning coalition can become losing in a coarser partition since other players forming coalitions might give a 'stronger resistance' against the winning coalition, or make the winning coalition more likely to 'break down'.

Plurality games and supporting weights We call a simple game in partition function form $v$ a plurality game if (i) it is monotonic, and (ii) for each $\pi \in \mathcal{P}$ we 
have $v(S ; \pi)=1$ for at least one $S \in \pi$. A plurality game $v$ is decisive, if for each $\pi \in \mathcal{P}$ we have that $v(S ; \pi)=1$ for exactly one $S \in \pi$. A plurality game $v$ is weighted, if there exists a weight vector $w \in X_{+}^{N}:=\left\{w \in \mathbb{R}_{+}^{N} \mid \Sigma_{i \in N} w_{i}=1\right\}$ such that for each $(S ; \pi) \in E,(S ; \pi) \in E_{W}(v)$ implies $w(S):=\Sigma_{i \in S} w_{i} \geq \Sigma_{i \in T} w_{i}:=w(T)$ for each $T \in \pi$. We call $w$ a supporting weight vector for the plurality game. In words, a plurality game is weighted if there exist nonnegative weights for the players, such that if an embedded coalition is winning, then the sum of the weights of the players in the winning coalition is maximal among the coalitions in the corresponding partition.

The example given below illustrates that a decisive plurality game need not be weighted.

Example 1 Consider the following five-player decisive plurality game, where we slightly abuse notation and write for instance $12,34,5$ to denote the partition $\{\{1,2\},\{3,4\},\{5\}\}$ with the coalition $\{1,2\}$ being winning in it, i.e., $12,34,5$ means that $v(\{1,2\} ;\{\{1,2\},\{3,4\},\{5\}\})=1$.

\begin{tabular}{|c|c|c|c|}
\hline 2345 & $234,1,5$ & $12,34,5$ & $15,24,3$ \\
\hline$\overline{1234,5}$ & $\underline{125}, 34$ & $\underline{12}, 3,45$ & $\underline{15}, 34,2$ \\
\hline$\overline{1235}, 4$ & $\overline{125}, 3,4$ & $\underline{12}, 35,4$ & $\underline{\overline{15}}, 2,3,4$ \\
\hline$\overline{1245}, 3$ & $\overline{135}, 24$ & $\overline{12}, 3,4,5$ & $\underline{25}, 34,1$ \\
\hline$\overline{1345}, 2$ & $\overline{135}, 2,4$ & $\overline{13}, 24,5$ & $\underline{25}, 1,3,4$ \\
\hline$\overline{2345}, 1$ & $\underline{235}, 14$ & $\underline{13}, 25,4$ & $\overline{24}, \underline{1}, 35$ \\
\hline$\overline{123,45}$ & $\overline{235}, 1,4$ & $\overline{13}, 45,2$ & $24, \overline{1}, 3,5$ \\
\hline $123,4,5$ & $\underline{145}, 23$ & $\underline{13}, 2,4,5$ & $\underline{23}, \overline{1}, 45$ \\
\hline$\overline{124}, 35$ & $\overline{145}, 2,3$ & $\overline{14}, \underline{23}, 5$ & $\underline{23}, 1,4,5$ \\
\hline$\overline{124}, 3,5$ & $\overline{245}, 13$ & $14, \overline{25}, 3$ & $\overline{35}, 1,2,4$ \\
\hline$\overline{134}, 25$ & $\underline{245}, 1,3$ & $\underline{14}, 35,2$ & $\underline{34}, \overline{1}, 2,5$ \\
\hline$\overline{134}, 2,5$ & $\overline{345}, 12$ & $\overline{14}, 2,3,5$ & $\overline{45}, 1,2,3$ \\
\hline$\overline{234}, 15$ & $\overline{345}, 1,2$ & $\underline{15}, 23,4$ & $\overline{1,2}, 3,4,5$ \\
\hline
\end{tabular}

Suppose that the above game were weighted with $w \in X_{+}^{N}$. We should then have $w_{4}+w_{5} \geq w_{1}$ (due to $\underline{45}, 1,2,3$ ) and $w_{1} \geq w_{2}+w_{4}$ (due to $24,1,3,5$ ) and thus, (by adding these two inequalities) $w_{5} \geq w_{2}$. On the other hand, from $w_{1} \geq w_{2}+w_{4}$ and $w_{2}+w_{3} \geq w_{1}$ (by $23,1,45$ ) follows $w_{3} \geq w_{4}$. Finally, from $w_{1} \geq w_{2}+w_{4}$ and $w_{2}+w_{5} \geq w_{1}$ (by $\underline{25}, 1, \overline{3}, 4$ ) we have $w_{5} \geq w_{4}$.

We also have $w_{3}+w_{4} \geq w_{1}$ (by $\underline{34}, 1,2,5$ ), and it follows with $w_{1} \geq w_{2}+w_{4}$ (see above) that $w_{3} \geq w_{2}$. We have $w_{1} \geq w_{3}+w_{5}$ (by 24, 1,35) and thus, with $w_{3}+w_{4} \geq w_{1}$ (by $\underline{34}, 1,2,5$ ), we have $w_{4} \geq w_{5}$. Summarizing, so far we have $w_{1} \geq w_{3} \geq w_{4}=w_{5} \geq w_{2}$.

Further, we have $w_{2}+w_{5} \geq w_{3}+w_{4}$ (by $25,34,1$ ) and thus, with $w_{4}=w_{5}$ and $w_{3} \geq w_{2}$ (see above), this gives $w_{2}=w_{3}$. Hence, $w_{1} \geq w_{3}=w_{4}=w_{5}=w_{2}$ should hold.

Moreover, from $w_{2}+w_{3} \geq w_{1}+w_{4}$ (by 14, 23, 5) and $w_{2}=w_{4}$, follows $w_{3} \geq w_{1}$. We conclude then that all weights should be equal. However, $w_{1} \geq w_{2}+w_{4}$ (by $24,1,35)$ then implies that $w_{1}=w_{2}=w_{4}=0$, which results in all weights being equal to zero. Thus, we have a contradiction to $w \in X_{+}^{N}$. 
In view of the above example, in what follows we first concentrate on decisive plurality games with at most four players (Sect. 3), and then consider additional restrictions on games with an arbitrary number of players guaranteeing that these games are weighted (Sects. 4 and 5).

\section{Games with at most four players}

We start our analysis by considering decisive plurality games with at most four players. A first reason to start by considering four player games, is that these are very illustrative, and hint to conditions for games with more than four players to be weighted.

A second reason to start with four player games is that, also for standard simple games (simple games without externalities), four player games received considerable attention. For instance, von Neumann and Morgenstern (1944) show that all simple games with less than four players, all proper or strong simple games with less than five players, and all constant sum games with less than six players have voting representations, i.e., they can be represented by weights assigned to the players. However, there are constant-sum games with six players for which representative weights do not exist. In their study of rough weightedness of small games, Gvozdeva and Slinko (2011) show that all games with at most four players, all strong or proper games with at most five players, and all constant-sum games with at most six players are roughly weighted. Finally, it is worth mentioning that Shapley (1962) also explicitly analyses games with four or less players when discussing several properties of weighted majority games. In particular, he studies homogeneous games where the weights can be assigned in such a way that all minimal winning coalitions have the same weight.

For decisive games with at most four players, it turns out that the winning coalition in a partition is either one of maximal size, or the one containing the winner in the atomistic partition. In our results, for convenience and without loss of generality, we will often assume that player 1 is the winner in the atomistic partition of a decisive plurality game.

Proposition 1 Let $v$ be a decisive plurality game with at most four players and $v\left(\{1\} ; \pi^{a}\right)=1$. Then for each $(S ; \pi) \in E,(S ; \pi) \in E_{W}(v)$ implies either $|S| \geq|T|$ for each $T \in \pi$, or $S=\pi(1)$.

The above conclusion is very helpful for the proof of our main result in this section.

Theorem 1 Every decisive plurality game with at most four players is weighted.

The proofs of Proposition 1 and Theorem 1 can be found in Appendix A. 


\section{Majority games and symmetric players}

Proposition 1 suggests that, if the winning coalition does not contain the winner in the atomistic partition, then it is only the size of a coalition that matters. For an arbitrary number of players, this fact invites us to consider the following type of majority games.

A plurality game $v$ is a majority game if for all $(S ; \pi) \in E$, every winning coalition is one of maximal size in the partition, i.e., $(S ; \pi) \in E_{W}(v)$ implies $|S| \geq|T|$ for each $T \in \pi$. It is easy to verify that $w=\left(\frac{1}{n}, \frac{1}{n}, \ldots, \frac{1}{n}\right)$ is a supporting weight vector for every majority game.

Theorem 2 Every majority game is weighted.

Of special interest are majority games where the players exhibit some kind of symmetry. For simple games in partition function form, several symmetry notions can be defined. Two possibilities are the following. Given a plurality game $v$, we say that two players $i, j \in N$ are ${ }^{2}$

- switch-symmetric in $v$, if for each $\pi \in \mathcal{P}$ with $j \notin \pi(i)$, $(\pi(i) ; \pi) \in E_{W}(v)$ implies that $\pi(i) \backslash\{i\} \cup\{j\}$ is winning in the partition $\left\{\pi(i) \backslash\{i\} \cup\{j\}, \pi(j) \backslash\{j\} \cup\{i\}, \pi_{N \backslash(\pi(i) \cup \pi(j))}\right\}$;

- grow-symmetric in $v$, if for each $\pi \in \mathcal{P}$ with $S, T \in \pi$ and $i, j \notin S \cup T$, $(S ; \pi) \in E_{W}(v)$ implies that $S \cup\{i\}$ is winning in the partition $\left\{S \cup\{i\}, T \cup\{j\}, \pi(i) \backslash\{i\}, \pi(j) \backslash\{j\}, \pi_{N \backslash(S \cup T \cup \pi(i) \cup \pi(j))}\right\}$.

Notice that in the definitions above, we can switch the roles of players $i$ and $j$. A plurality game $v$ is switch-symmetric (respectively grow-symmetric) if all players ${ }^{3}$ are switch-symmetric (respectively grow-symmetric) in $v$.

Each of these symmetry notions expresses an independence idea. Two players being switch-symmetric in a game requires that a winning coalition that contains one of them but not the other player, remains winning in the partition obtained by exchanging the places of these two players. On the other hand, grow-symmetry requires that for a winning and a losing coalition not containing both players, the winning coalition should remain winning if we add either one of these players to the winning, and the other to the losing coalition.

Our next result provides a characterization of switch-symmetric majority games and shows that, on the class of majority games, switch-symmetry implies grow-symmetry. ${ }^{4}$

\footnotetext{
${ }^{2}$ For notational convenience, we are not quite precise here since, in case $\pi(i) \backslash\{i\}$ or $\pi(j) \backslash\{j\}$ is the empty set, then this set should be deleted from the partition.

${ }^{3}$ This means that each pair of players is switch-symmetric (respectively grow-symmetric).

${ }^{4}$ We use symmetry among players in order to introduce classes of decisive plurality games, i.e., we impose these conditions on games. Although it is worthwhile to study properties of the symmetry relation itself, for example to see if these notions define equivalence classes, this is beyond the goal of this paper. Proposition 2, in combination with Example 2 shows that, on the class of majority games, there
} 
Proposition 2 A majority game is switch-symmetric if and only if all coalitions of maximal size in a partition are winning in it. Moreover, any switch-symmetric majority game is also grow-symmetric.

The proofs of this section can be found in Appendix B.

It is worth mentioning that not every grow-symmetric majority game is also switch-symmetric, as illustrated by the following example.

Example 2 Consider the four-player decisive majority game $v$ defined below.

$\begin{array}{lll}\frac{1234}{123}, 4 & \frac{12}{12}, 34 & \underline{14}, 2,3 \\ \frac{124}{12}, 3 & \frac{13}{13}, 24 & \underline{24}, 1,3 \\ \frac{134}{13}, 2 & \underline{13}, 2,4 & \underline{34}, 1,2 \\ \underline{234}, 1 & \underline{14}, 23 & \underline{1}, 2,3,4\end{array}$

Notice first that all players from $N \backslash\{1\}$ are grow-symmetric in $v$. To see this, take, for example, players 2 and 3, and a partition with two coalitions, say $S$ and $T$, that (i) do not contain these two players $(2,3 \notin S \cup T)$, and (ii) are such that one coalition, say $S$, is winning, and the other coalition $T$ is losing in the partition. In fact, the only partition of this type is the atomistic partition with $\left(\{1\} ; \pi^{a}\right) \in E_{W}(v)$. Since the coalition $\pi(1)$ containing player 1 is winning in any partition consisting of two two-player coalitions, irrespective of which player joins player 1, players 2 and 3 are grow-symmetric in $v$. A similar argument for player 4 shows that all players in $N \backslash\{1\}$ are grow-symmetric in $v$. To see that player 1 is also grow-symmetric with the other players in $v$, take player 1 and, for example, player 2 . Notice that there is no partition with at least two coalitions that do not contain players 1 and 2, such that one of these two coalitions is winning. Therefore, players 1 and 2, and thus all players, are obviously grow-symmetric in $v$. However, from the fact that $\{1\}$ is the unique winning coalition in the atomistic partition, it immediately follows that the game is not switch-symmetric.

If we require a majority game to be switch-symmetric, then the equal weight vector used to prove Theorem 2 , is the only one making the game weighted.

Proposition 3 If a weight vector supports a switch-symmetric majority game, then all weights are equal in this weight vector.

As one can see, this result is clearly driven by the fact that, in a switch-symmetric majority game, all largest coalitions in a partition are winning in it.

Footnote 4 (continued)

is an implication only in one direction. Examples 3 and 4 (in Sect. 5) show that, on the class of $(n-1)$ -symmetric games, the above two statements are independent. 


\section{$5(n-1)$-symmetric games}

We have defined decisive plurality games as games where there is exactly one winning coalition in each partition. Since this implies that there is a unique winner in the atomistic partition, no decisive plurality game is switch-symmetric. The 'most symmetric' a game can be is if all other players are symmetric. Therefore, we call a decisive plurality game $(n-1)$ - switch-symmetric if all players but one (the winner in the atomistic partition) are switch-symmetric in the game. We study the impact of this restriction on the game's support. Due to the decisiveness of $v$, the following result is immediate.

Proposition 4 Let $v$ be a decisive plurality game with at least three players and $v\left(\{1\} ; \pi^{a}\right)=1$. If $v$ is $(n-1)$-switch-symmetric, then all players in $N \backslash\{1\}$ are switch-symmetric in $v$.

Recall from Proposition 2, that for the case of majority games, grow-symmetry is implied by switch-symmetry. We define a decisive plurality game $v$ to be $(n-1)$ -grow-symmetric if all players but one (the winner in the atomistic partition) are grow-symmetric in $v$, and show that $(n-1)$-switch-symmetry and ( $n-1)$-growsymmetry are independent properties.

For this, let us first show that $(n-1)$-switch-symmetry does not imply $(n-1)$ -grow-symmetry.

Example 3 Consider the six-player $(n-1)$-switch-symmetric decisive game $v$ with player set $N=\{1, i, j, k, \ell, m\}$ and coalition $\pi(1)$ winning in every partition $\pi \in \mathcal{P}$, except in the partitions of type $\{\{1\},\{i, j, k\},\{\ell, m\}\},\{\{1\},\{i, j, k\},\{\ell\},\{m\}\}$, $\{\{1\},\{i, j, k, \ell\},\{m\}\}$, and $\{\{1\},\{i, j, k, \ell, m\}\}$ where it is always the largest coalition in the partition that wins. The game is indeed $(n-1)$-switch-symmetric since exchanging any two players from $N \backslash\{1\}$ does not change the size of the (corresponding) winning coalition in a partition. Suppose now that the game $v$ is $(n-1)$ -grow-symmetric and consider for instance players $\ell$ and $m$. Then $1, i j k, \ell m$ should imply that $1 m$, ijk $\ell$ which is in contradiction to the fact that $\{1, m\}$ wins in the partition $\{\{1, m\},\{i \overline{i, j, k}, \ell\}\}$.

Our next example shows that $(n-1)$-grow-symmetry does not imply $(n-1)$ -switch-symmetry.

Example 4 Consider the five-player decisive majority game $v$ on $N=\{1,2,3,4,5\}$ where, for every partition $\pi \in \mathcal{P}$ containing exactly two coalitions $S$ and $T$ of maximal size, we have that $1 \in S \cup T$ implies $(\pi(1) ; \pi) \in E_{W}(v)$, while $1 \notin S \cup T$ implies $(\pi(2) ; \pi) \in E_{W}(v)$. Assume additionally that $\left(\{1\} ; \pi^{a}\right) \in E_{W}(v)$. Obviously, players 3,4 and 5 are grow-symmetric in $v$. To show that players 2 and 3 are also growsymmetric in $v$, notice that the only partitions containing two coalitions that do not contain either of these two players, such that one of these coalitions is winning, are $1, \underline{45}, 2,3 ; 4, \underline{15}, 23 ; 4, \underline{15}, 2,3 ; 5, \underline{14}, 23 ; 5, \underline{14}, 2,3$; and the atomistic partition. By 
majority, the winning coalition in these partitions stays winning after either player 2 or player 3 joins, showing that players 2 and 3, and thus all players in $N \backslash\{1\}$ are grow-symmetric in $v$; thus, the game is $(n-1)$-grow-symmetric. Notice, however, that the game is not $(n-1)$-switch-symmetric since, considering players 2 and 4 , we have that $\{2,5\}$ wins in $\{\{2,5\},\{3,4\},\{1\}\}$ but $\{4,5\}$ loses in $\{\{2,3\},\{4,5\},\{1\}\}$.

In what follows, we call a decisive plurality game $v$

- $(n-1)$-symmetric, if all players but the winner in the atomistic partition are both switch- and grow-symmetric in $v$.

$(n-1)$-symmetric decisive plurality games turn out to be weighted (Theorem 3 ). We show this by proving important implications (stated in Propositions 4-6) on the winning embedded coalitions in such games. In these results, we also explicitly state the type of $(n-1)$-symmetry (i.e., $(n-1)$-symmetry, $(n-1)$-switchsymmetry, or $(n-1)$-grow-symmetry) which is used in the corresponding proofs. All proofs of this section are provided in Appendix C.

We start by showing that in $(n-1)$-switch-symmetric games, a winning coalition in a partition either contains the winner in the atomistic partition, or has strictly more players than any other coalition in the partition.

Proposition 5 Let $v$ be a decisive and $(n-1)$-switch-symmetric plurality game with $v\left(\{1\} ; \pi^{a}\right)=1$. Then $(S ; \pi) \in E_{W}(v)$ implies either $S=\pi(1)$ or $|S|>|T|$ for each $T \in \pi \backslash\{S, \pi(1)\}$.

This proposition gives as a corollary that in a partition having two or more coalitions of maximal size, the winning coalition should contain the winner in the atomistic partition.

Corollary 1 Let $v$ be a decisive and $(n-1)$-switch-symmetric plurality game with $v\left(\{1\} ; \pi^{a}\right)=1$, and let $\pi \in \mathcal{P}$. If $\pi \backslash\{\pi(1)\}$ contains at least two largest coalitions, then $(\pi(1) ; \pi) \in E_{W}(v)$.

Define $\mathcal{P}_{1}=\{\pi \in \mathcal{P} \mid\{1\} \in \pi\}$ as the collection of those partitions that contain singleton $\{1\}$. In what follows, we make use of the power $p_{1}(v)$ of player 1 in a decisive plurality game $v$ with $v\left(\{1\} ; \pi^{a}\right)=1$, and define it as

$$
p_{1}(v)=\max _{\pi \in \mathcal{P}_{1}}\left\{t \mid \exists T \in \pi \text { with }|T|=t \text { and }(\{1\} ; \pi) \in E_{W}(v)\right\},
$$

that is, it is the size of a largest coalition that loses against $\{1\}$ in some partition from $\mathcal{P}_{1}$. We denote by $\mathcal{P}^{*}$ the set of all partitions in which there is only one largest coalition that does not contain player 1 . For $\pi \in \mathcal{P}^{*}, S_{\pi}$ stands for the largest coalition in $\pi \backslash\{\pi(1)\}$.

Our next result explains the crucial role of $p_{1}(v)$ in determining the winning coalitions in the partitions contained in $\mathcal{P}^{*}$. 
Proposition 6 Let $v$ be a decisive and $(n-1)$-symmetric plurality game with at least five players and $v\left(\{1\} ; \pi^{a}\right)=1$, and let $\pi \in \mathcal{P}^{*}$. Then,

1. $\left|S_{\pi}\right| \geq p_{1}(v)+|\pi(1)|$ implies $\left(S_{\pi} ; \pi\right) \in E_{W}(v)$;

2. $\left|S_{\pi}\right|<p_{1}(v)+|\pi(1)|$ implies $(\pi(1) ; \pi) \in E_{W}(v)$.

Corollary 1 and Proposition 6 allow for a complete characterization of the winning embedded coalitions in ( $n-1)$-symmetric games. For $\pi \in \mathcal{P}$, the coalition $\pi(1)$ is winning in $\pi$ when either $\pi \backslash\{\pi(1)\}$ contains at least two largest coalitions, or the unique largest coalition in $\pi \backslash\{\pi(1)\}$ is smaller than the threshold level $p_{1}(v)+|\pi(1)|$. Otherwise, it is the unique largest coalition which wins in the partition $\pi$. Notice that, in the two cases where $\pi(1)$ is winning in $\pi$, a coalition $S \in \pi$ with $|S|>|\pi(1)|$ may exist; so, it is not necessarily that a largest coalition wins in a partition.

The value $p_{1}(v)$ also plays an important role in the following main result on $(n-1)$-symmetric plurality games, which gives explicit supporting weights for a decisive $(n-1)$-symmetric plurality game.

Theorem 3 Let $v$ be a decisive and $(n-1)$-symmetric plurality game with $v\left(\{1\} ; \pi^{a}\right)=1$. Then $v$ is weighted. In particular, the weight vector $w$ defined by $w_{1}=\frac{p_{1}(v)}{p_{1}(v)+|N|-1}$ and $w_{i}=\frac{1}{p_{1}(v)+|N|-1}$ for each $i \in N \backslash\{1\}$ supports $v$.

The following example sheds light on how the results in this section lead to a plurality game being weighted.

Example 5 Consider a five-player decisive and $(n-1)$-symmetric game $v$ with $v\left(\{1\} ; \pi^{a}\right)=1$. Notice first that it is impossible for the power of player 1 in the game $v$ to be $p_{1}(v)=1$. The reason is that $p_{1}(v)=1$ would imply that in the partition $\{\{1\},\{2,3\},\{4,5\}\}$ either $\{2,3\}$ or $\{4,5\}$ should be winning, which is a contradiction to Corollary 1 . So, $p_{1}(v) \in\{2,3,4\}$.

If $p_{1}(v)=4$, then $(\{1\} ;\{\{1\},\{2,3,4,5\}\}) \in E_{W}(v)$ and thus, by monotonicity, the winning coalition in any partition contains player 1 . Clearly then, the vector $w=\left(\frac{1}{2}, \frac{1}{8}, \frac{1}{8}, \frac{1}{8}, \frac{1}{8}\right)$ supports the game $v$.

Suppose next that $p_{1}(v)=3$ holds. Since $p_{1}(v)+|\pi(1)| \geq 4$, we have, by Corollary 1 and Proposition 6 , that $\pi(1)$ is winning in all partitions except in $\{\{1\},\{2,3,4,5\}\}$ (where $\{2,3,4,5\}$ is winning). It can be checked that the vector $w=\left(\frac{3}{7}, \frac{1}{7}, \frac{1}{7}, \frac{1}{7}, \frac{1}{7}\right)$ supports the game $v$.

Finally, consider the case of $p_{1}(v)=2$. Due to $p_{1}(v)+|\pi(1)| \geq 3$, we have, by Corollary 1 and Proposition 6 , that $\pi(1)$ is winning in all partitions except in $\{\{1\},\{i, j, k\},\{\ell\}\}$ (where $\{i, j, k\}$ is winning) and $\{\{1\},\{i, j, k, \ell\}\}$ (where $\{i, j, k, \ell\}$ is winning). Clearly then, the weight vector $w=\left(\frac{1}{3}, \frac{1}{6}, \frac{1}{6}, \frac{1}{6}, \frac{1}{6}\right)$ supports the game $v$.

Let us finally remark that decisive plurality games are always weighted in case there are no externalities. We say that a plurality game $v$ is externality-free if $v(S ; \pi)=v\left(S ; \pi^{\prime}\right)$ holds for all $S \subseteq N$ and for all partitions $\pi, \pi^{\prime} \in \mathcal{P}$ such that $S \in \pi \cap \pi^{\prime}$. 
Proposition 7 Every externality-free, decisive plurality game is weighted.

This proposition makes clear that problems with decisive plurality games being not weighted arise from the existence of (negative) externalities.

\section{Related literature}

In our study about the possibility of assigning weights to players in simple partition function form games, we have presented classes of games which do allow for a weighted representation. Moreover, the support of the corresponding games was shown to crucially shape the set of possible winning coalitions in a partition and thus, to shed light on which coalitions are most powerful in the presence of (negative) externalities. This naturally places our work within the strands of literature devoted to the numerical representation of standard simple games as well as to the study of general partition function form games.

The first strand of literature is mainly concerned with the question whether it is (always) possible to represent a standard simple game as a weighted majority game, that is, to find non-negative weights and a positive real number (quota) such that a coalition is winning in the simple game if and only if the combined weights of its members weakly exceeds the quota. As shown by von Neumann and Morgenstern (1944), not all simple games do allow for such weighted majority representation. The question of finding properties that characterize weighted games within the class of simple games was then naturally posted by Isbell (1956, (1958), and answered by Elgot (1961) and Taylor and Zwicker (1992). More precisely, Taylor and Zwicker (1992) characterize weighted voting in terms of the ways in which coalitions can gain or lose by trading among themselves, while Hammet et al. (1981) and Einy and Lehrer (1989) answer the above question by using results about separating convex sets. Peleg (1968) and Sudhölter (1996) show for the case of (constant-sum) weighted majority games that, correspondingly, the nucleolus and the modified nucleolus induce a representation of the game.

If a standard simple game does not allow for a weighted representation, then one might consider rough weights (cf. Taylor and Zwicker 1999). A simple game is roughly weighted if there exist weights and a threshold such that every coalition with the sum of its players' weights being above (respectively below) the threshold is winning (respectively losing). Again, not all standard simple games turn out to be roughly weighted. Gvozdeva and Slinko (2011) give necessary and sufficient conditions for a simple game to have rough weights. Related to the issue of non-weightedness of games, Carreras and Freixas (1996) and Freixas and Molinero (2009) investigate complete simple games being simple games behaving in some respects as weighted simple games.

All the papers cited above deal with standard simple games, while the focus of our work is on the weighted representation of plurality games which we defined as special type of simple games in partition function form. To the best of our knowledge, we are the first to study and provide conditions assuring weighted representations of such games. 
The second strand of related literature deals with general partition function form games as initiated by the seminal works of Thrall (1962) and Thrall and Lucas (1963), and recently surveyed by Koczy (2018). Besides the investigation of general properties of such games (e.g., Lucas and Marcelli 1978; Maskin 2003; Hafalir 2007), there are two main issues of simultaneous interest in the corresponding works: which coalitions will form (cf. Ray 2007), and how the coalitional worths will be allocated to their members. For instance, de Clippel and Serrano (2008) separate the intrinsic payoffs from those due to the externalities of coalition formation. However, the main focus in that literature has been on extending the Shapley value for games with externalities (e.g., Myerson 1977; Gilboa and Lehrer 1991; Albizuri et al. 2005; Macho-Stadler et al. 2007, de Clippel and Serrano (2008); McQuillin (2009); Dutta et al. (2010); Grabisch and Funaki (2012)) and on extending different power indices to the class of simple games with externalities (e.g., Bolger 1986; Alonso-Meijide et al. 2017; Alvarez Mozos et al. 2017).

Although the study of power indices for simple partition function form games was out of the scope of this paper, we nevertheless used a notion of power (for the winner in the atomistic partition) in order to derive our results with respect to $(n-1)$ -symmetric plurality games. In follow-up research, we intend to generalize this notion as to apply to each player, to investigate in detail its properties, and to axiomatically characterize it.

Funding Open Access funding enabled and organized by Projekt DEAL.

Open Access This article is licensed under a Creative Commons Attribution 4.0 International License, which permits use, sharing, adaptation, distribution and reproduction in any medium or format, as long as you give appropriate credit to the original author(s) and the source, provide a link to the Creative Commons licence, and indicate if changes were made. The images or other third party material in this article are included in the article's Creative Commons licence, unless indicated otherwise in a credit line to the material. If material is not included in the article's Creative Commons licence and your intended use is not permitted by statutory regulation or exceeds the permitted use, you will need to obtain permission directly from the copyright holder. To view a copy of this licence, visit http://creativecommons.org/licen ses/by/4.0/.

\section{Appendix: Proofs from Sect. 3}

Proof of Proposition 1 Notice first that for $|N| \leq 3$ the assertion follows by $\{1\}$ being winning in the atomistic partition and the monotonicity of $v$. Suppose now that $|N|=4$, i.e., $N=\{i, j, k, \ell\}$, and let there exist $(S ; \pi) \in E$ with $(S ; \pi) \in E_{W}(v)$, $1 \notin S$, and $|S|<|T|$ for some $T \in \pi$. We show that this leads to a contradiction. Since there are only four players, we have $|S|=1$. Assume, w.l.o.g., that $(S ; \pi)=(\{i\} ;\{\{i\},\{j\},\{k, \ell\}\})$ with $i \neq 1$ holds. Then, by monotonicity of $v$, $(\{i\} ;\{\{i\},\{j\},\{k\},\{\ell\}\}) \in E_{W}(v)$ should hold as well, giving a contradiction to $v$ being decisive and $\{i\} \neq\{1\}$. By a similar argument, it can be shown that $(S ; \pi) \in E_{W}(v)$ cannot be of the form $\{\{i\} ;\{\{i\},\{j, k, \ell\}\}\}$ with $i \neq 1$.

Proof of Theorem 1 Let $v$ be a decisive plurality game defined on the player set $N$ and assume that, w.l.o.g., player 1 wins in the atomistic partition. For $|N|=2$, the vector $w=\left(\frac{1}{2}, \frac{1}{2}\right)$ supports the game $v$. 
Table 1 Proof of Theorem 1, Case 2

\begin{tabular}{|c|c|c|}
\hline $1 j k \ell$ & $1 j, k \ell$ & $1, \ell, j k$ \\
\hline$\overline{\overline{1 k \ell}}, j$ & $\overline{1 j}, k, \ell$ & $\underline{1 k, j, \overline{\bar{\ell}}}$ \\
\hline$\overline{1 j \ell, k}$ & $\overline{\overline{1 k}}, \underline{j \ell}$ & $\underline{\overline{1 \ell}}, j, k$ \\
\hline$\overline{1 j k}, \ell$ & $1, k, j \ell$ & $\overline{1, j, k \ell}$ \\
\hline$\overline{j k t}, 1$ & $1 \ell, \overline{j k}$ & $\underline{1}, j, k, \ell$ \\
\hline
\end{tabular}

If $|N|=3$, let us take $N=\{1, j, k\}$. By monotonicity of $v$, $(\{1, j\} ;\{\{1, j\},\{k\}\}) \in E_{W}(v)$ and $(\{1, k\} ;\{\{1, k\},\{j\}\}) \in E_{W}(v)$ holds. Take the weight vector $w$ with $w_{1}=\frac{1}{2}$ and $w_{j}=w_{k}=\frac{1}{4}$. Notice that it supports $v$ independently of the fact whether coalition $\{1\}$ or coalition $\{j, k\}$ is winning in the partition $\{\{1\},\{j, k\}\}$.

Finally, if $|N|=4$ let us take $N=\{1, j, k, \ell\}$. Since player 1 is winning in the atomistic partition, we have that if supporting weights $w$ do exist, it must hold that $w_{1} \geq w_{j}, w_{k}, w_{\ell}$. We distinguish the following cases with respect to bipartitions containing coalitions of size 2 .

Case 1 (Player 1 is in no winning coalition in a bipartition of this type). Let $v$ be such that $(\{k, \ell\} ;\{\{1, j\},\{k, \ell\}\}) \in E_{W}(v),(\{j, \ell\} ;\{\{1, k\},\{j, \ell\}\}) \in E_{W}(v)$, and $(\{j, k\} ;\{\{1, \ell\},\{j, k\}\}) \in E_{W}(v)$. Take the vector $w=\left(\frac{1}{4}, \frac{1}{4}, \frac{1}{4}, \frac{1}{4}\right)$. Notice that it supports $v$ for the winning coalitions in the above partitions. Further, by monotonicity of $v,(\{k, \ell\} ;\{\{1\},\{j\},\{k, \ell\}\}) \in E_{W}(v), \quad(\{j, \ell\} ;\{\{1\},\{k\},\{j, \ell\}\}) \in E_{W}(v)$, and $(\{j, k\} ;\{\{1\},\{\ell\},\{j, k\}\}) \in E_{W}(v)$. Clearly, $w$ is a suitable weight vector for these embedded coalitions as well. Again by monotonicity of $v$, $(\{j, k, \ell\} ;\{\{1\},\{j, k, \ell\}\}) \in E_{W}(v)$ and weight vector $w$ still works here. We conclude that weight vector $w$ indeed supports game $v$.

Case 2 (Player 1 is in the winning coalition of one bipartition of this type). Let $v$ be such that $(\{1, j\} ;\{\{1, j\},\{k, \ell\}\}) \in E_{W}(v),(\{j, \ell\} ;\{\{1, k\},\{j, \ell\}\}) \in E_{W}(v)$, and $(\{j, k\} ;\{\{1, \ell\},\{j, k\}\}) \in E_{W}(v)$. The vector $w$ with $w_{1}=w_{j}=\frac{1}{3}>\frac{1}{6}=w_{k}=w_{\ell}$ works for these winning embedded coalitions. It can be checked that $w$ is a suitable weight vector also in the embedded coalitions $(S ;\{\{k, \ell\},\{1\},\{j\}\})$, irrespective of the winning coalition $S \in\{\{k, \ell\},\{1\},\{j\}\}$. The winning coalitions in the other partitions are determined by monotonicity of the game as displayed in Table 1 . In this table, the single underlined coalitions are winning in the corresponding partitions by assumption, and the double underlined coalitions are winning by monotonicity. A partition with no underlined coalition displays the fact that the assumptions do not exactly determine which coalition is winning.

Case 3 (Player 1 is in the winning coalition of two bipartitions of this type). Let $v$ be such that $(\{1, j\} ;\{\{1, j\},\{k, \ell\}\}) \in E_{W}(v),(\{1, k\} ;\{\{1, k\},\{j, \ell\}\}) \in E_{W}(v)$, and $(\{j, k\} ;\{\{1, \ell\},\{j, k\}\}) \in E_{W}(v)$. Take the weight vector $w$ with $w_{1}=w_{j}=w_{k}=\frac{1}{3}>0=w_{\ell}$. It can be checked that $w$ is a suitable weight vector, also irrespective of the winning coalitions in the partitions $\{\{j, \ell\},\{1\},\{k\}\}$ and 
Table 2 Proof of Theorem 1, Case 3

\begin{tabular}{lll}
\hline$\underline{1 j k \ell}$ & $\underline{1 j}, k \ell$ & $1, \ell, j k$ \\
$\overline{\overline{1 k \ell}, j}, j$ & $\underline{\overline{1 j}}, k, \ell$ & $\underline{\overline{1 k}, j, \overline{\bar{\ell}}}$ \\
$\overline{\overline{1 j \ell}, k}, k$ & $\underline{\overline{1 k}}, j \ell$ & $\underline{\underline{1 \ell}}, j, k$ \\
$\overline{\underline{1 j k}, \ell}$ & $1, k, j \ell$ & $1, j, k \ell$ \\
$\overline{\overline{\overline{k k \ell}}, 1}$ & $1 \ell, j k$ & $\underline{\underline{1}}, j, k, \ell$ \\
\hline
\end{tabular}

Table 3 Proof of Theorem 1, Case 4.1

\begin{tabular}{|c|c|c|}
\hline $1 j k \ell$ & $1 j, k \ell$ & $1, \ell, j k$ \\
\hline$\overline{\overline{\overline{k \ell}}}, j$ & $\underline{1 j}, k, \ell$ & $\underline{\underline{1 k}}, j, \ell$ \\
\hline$\underline{1 j \ell, k}$ & $\underline{\overline{1 k}}, j \ell$ & $\underline{\underline{1 \ell}}, j, k$ \\
\hline$\overline{1 j k}, \ell$ & $1, k, j \ell$ & $\overline{1, j, k \ell}$ \\
\hline$\overline{\overline{j k \ell}}, 1$ & $\underline{1 \ell}, j k$ & $\underline{1}, j, k, \ell$ \\
\hline
\end{tabular}

Table 4 Proof of Theorem 1, Case 4.2

\begin{tabular}{lll}
\hline$\frac{1 j k \ell}{\overline{\overline{1 k \ell}}, j}, k$ & $\underline{1 j}, k \ell, \ell k$ \\
$\overline{\overline{1 j \ell}, k}, k, \ell$ & $\underline{\overline{1}}, j, \ell$ \\
$\overline{\overline{1 j k}}, \ell$ & $\underline{\overline{1 k}}, j \ell$ & $\underline{\overline{1 \ell}}, j, k$ \\
$\overline{\overline{j k \ell},}, 1$ & $\underline{1}, k, j \ell$ & $\underline{1}, j, k \ell$ \\
\hline
\end{tabular}

$\{\{k, \ell\},\{1\},\{j\}\}$. The winning coalitions in the other partitions are determined by monotonicity of the game, see Table 2.

Case 4 (Player 1 is in the winning coalition of all (three) bipartitions of this type). Let $v$ be such that $(\{1, j\} ;\{\{1, j\},\{k, \ell\}\}) \in E_{W}(v),(\{1, k\} ;\{\{1, k\},\{j, \ell\}\}) \in E_{W}(v)$, and $(\{1, \ell\} ;\{\{1, \ell\},\{j, k\}\}) \in E_{W}(v)$. Two sub-cases have to be considered:

(4.1) $(\{j, k, \ell\} ;\{\{1\},\{j, k, \ell\}\}) \in E_{W}(v)$. Take the vector $w$ with $w_{1}=\frac{2}{5}$ and $w_{j}=w_{k}=w_{\ell}=\frac{1}{5}$. It can be checked that $w$ is a suitable weight vector, also in the embedded coalitions with partitions $\{\{j, k\},\{1\},\{\ell\}\},\{\{j, \ell\},\{1\},\{k\}\}$, and $\{\{k, \ell\},\{1\},\{j\}\}$, irrespective of the winning coalitions in these partitions; notice that, due to Proposition 1 , there is no winning singleton in these partitions which differs from $\{1\}$. The winners in the other partitions are determined by monotonicity of the game, see Table 3.

(4.2) $(\{1\} ;\{\{1\},\{j, k, \ell\}\}) \in E_{W}(v)$. Then, by monotonicity, in every partition the winning coalition is the one containing player 1 . Take the weight vector $w$ with $w_{1}=1>0=w_{j}=w_{k}=w_{\ell}$. It can be checked that $w$ is a suitable weight vector by just applying the monotonicity of the game, see Table 4. 


\section{Appendix: Proofs from Sect. 4}

Proof of Proposition 2 We first show that if in a majority game $v$ all coalitions of maximal size in a partition are winning in it, then the game is switch-symmetric. Suppose that, on the contrary, there were two players, $i$ and $j$, who are not switch-symmetric in $v$. In such a case, there should be $S \subset N$ with $i \in S$ and $j \notin S$ such that $(S ; \pi) \in E_{W}(v)$, but $S \backslash\{i\} \cup\{j\}$ is not winning in the partition $\left\{S \backslash\{i\} \cup\{j\}, \pi(j) \backslash\{j\} \cup\{i\}, \pi_{N \backslash(S \cup \pi(j))}\right\}$. Since $S$ and $S \backslash\{i\} \cup\{j\}$ are of the same size, by $v$ being a majority game, $S$ is a largest coalition in $\pi$, and thus $S \backslash\{i\} \cup\{j\}$ is a largest coalition in $\left\{S \backslash\{i\} \cup\{j\}, \pi(j) \backslash\{j\} \cup\{i\}, \pi_{N \backslash(S \cup \pi(j))}\right\}$. By supposition, $(S \backslash\{i\}) \cup\{j\}$ should be winning in that partition, which gives a contradiction.

Let us show next that if a majority game $v$ is switch-symmetric, then all coalitions of maximal size in a partition are winning in it. Notice first that, by the definition of a majority game, a winning coalition in a partition should be of maximal size. We are left to show that all coalitions of maximal size are winning. For this, take $(S ; \pi) \in E_{W}(v)$ and suppose that there is $T \in \pi \backslash\{S\}$ with $|T|=|S|$. Since the game is switch-symmetric, we can (repeatedly) replace all players from $S \backslash T$ by those from $T \backslash S$ and conclude that $(T ; \pi) \in E_{W}(v)$ should hold.

Finally, to show that every switch-symmetric majority game is also growsymmetric, consider a switch-symmetric majority game $v$ and let us show that the game is also grow-symmetric. Take $i, j \in N$, and let $\pi \in \mathcal{P}$ with $S, T \in \pi$ be such that $(S ; \pi) \in E_{W}(v)$ and $i, j \notin S \cup T$. Consider then the partition $\pi^{\prime}=\left\{S \cup\{i\}, T \cup\{j\}, \pi_{N \backslash(S \cup T \cup\{i, j\})}\right\}$. If $S$ is the unique largest coalition in $\pi$, then $S \cup\{i\}$ will be the unique largest coalition also in $\pi^{\prime}$. By the definition of a majority game, $\left(S \cup\{i\} ; \pi^{\prime}\right) \in E_{W}(v)$. Suppose now that $S$ and $T$ are two largest coalitions in $\pi$. In such a case, $S \cup\{i\}$ and $T \cup\{j\}$ will be two largest coalitions also in $\pi^{\prime}$. Since the majority game $v$ is switch-symmetric, it follows from the above characterization of such games that all largest coalitions in $\pi^{\prime}$ are winning in it, i.e., $\left(S \cup\{i\} ; \pi^{\prime}\right) \in E_{W}(v)$. We conclude that the game $v$ is grow-symmetric as well.

Proof of Proposition 3 Suppose that a switch-symmetric majority game $v$ is supported by a weight vector $w$. Consider the atomistic partition $\pi^{a}$ and notice that, by Proposition 2, $\left(\{i\} ; \pi^{a}\right) \in E_{W}(v)$ holds for each $i \in N$. Since the game $v$ is weighted, we have that the inequalities $w_{k} \geq w_{\ell}$ and $w_{\ell} \geq w_{k}$ hold for all $k, \ell \in N$. We conclude then that all weights should be equal.

\section{Appendix: Proofs from Sect. 5}

Proof of Proposition 4 Since $\{1\}$ is the unique winning coalition in the atomistic partition, player 1 cannot be switch-symmetric in $v$ with any other player due to the decisiveness of the game. By definition of $(n-1)$-switch-symmetry of $v$, all players in $N \backslash\{1\}$ are switch-symmetric in $v$. 
Proof of Proposition 5 Take $(S ; \pi) \in E_{W}(v)$ and suppose that $S \neq \pi(1)$. Notice that $|S|>1$ should hold; otherwise, by monotonicity, the single player in $S$ should be winning in the atomistic partition, which is in contradiction to the decisiveness of $v$ and $\{1\}$ being the winner in the atomistic partition. We now have to show that $|T| \geq|S|$ for some $T \in \pi \backslash\{S, \pi(1)\}$, leads to a contradiction.

Suppose that such a coalition $T$ exists. Take $T^{\prime} \subseteq T$ with $\left|T^{\prime}\right|=|S|$. Since the game is $(n-1)$-switch-symmetric (and all players in $S \cup T$ are switch-symmetric in $v$ since both coalitions do not contain player 1), we can (repeatedly) replace all players from $S$ by those from $T^{\prime}$ and conclude that $\left(T^{\prime} ;\left\{T^{\prime}, S \cup\left(T \backslash T^{\prime}\right), \pi_{N \backslash(S \cup T)}\right\}\right) \in E_{W}(v)$. By monotonicity, $\left(T ;\left\{T, S, \pi_{N \backslash(S \cup T)}\right\}\right)=(T ; \pi) \in E_{W}(v)$ should hold as well, which is a contradiction to $(S ; \pi) \in E_{W}(v)$ and the decisiveness of the game.

The following two lemmas will be used in the proofs of Proposition 6 and Theorem 3.

Lemma 1 Let $v$ be a decisive and $(n-1)$-switch-symmetric plurality game with at least five players and $v\left(\{1\} ; \pi^{a}\right)=1$. Then $p_{v}(1) \in\left\{\frac{|N|-1}{2}, \ldots,|N|-1\right\}$ if $|N|$ is odd, and $p_{v}(1) \in\left\{\frac{|N|-2}{2}, \ldots,|N|-1\right\}$ if $|N|$ is even.

Proof of Lemma 1 Clearly, the largest coalition that could lose against $\{1\}$ in a partition is $N \backslash\{1\}$. Suppose first that $|N|$ is odd and thus, there exists a partition $\pi=\{\{1\}, S, T\}$ with $|S|=|T|=\frac{|N|-1}{2}$. By Corollary $1, \quad(\{1\} ; \pi) \in E_{W}(v)$, and thus, $p_{v}(1) \geq \frac{|N|-1}{2}$ should hold. If $|N|$ is even, one can take the partition $\pi^{\prime}=\left\{\{1\}, S^{\prime}, T^{\prime},\{i\}\right\}^{2}$ with $\left|S^{\prime}\right|=\left|T^{\prime}\right|=\frac{|N|-2}{2}$ as to conclude again from Corollary 1 that $p_{v}(1) \geq \frac{|N|-2}{2}$ must be the case.

Lemma 2 Let $v$ be a decisive and $(n-1)$-switch-symmetric plurality game with at least five players and $v\left(\{1\} ; \pi^{a}\right)=1$, and let $\pi \in \mathcal{P}^{*}$. Then $\left|S_{\pi}\right| \geq p_{1}(v)$ implies $|S| \leq p_{1}(v)$ for each $S \in \pi \backslash\left\{\pi(1), S_{\pi}\right\}$.

Proof of Lemma 2 Suppose not, i.e., $\left|S_{\pi}\right| \geq p_{1}(v)$ and there exists $S \in \pi \backslash\left\{\pi(1), S_{\pi}\right\}$ such that $|S|>p_{1}(v)$. If $|N|$ is odd, then $\left|S_{\pi}\right|+|S|>2 p_{1}(v) \geq|N|-1$ where the second inequality follows from Lemma 1 . Since $1 \notin S_{\pi} \cup S$, we have a contradiction. If $|N|$ is even, then $\left|S_{\pi}\right|+|S|>2 p_{1}(v) \geq|N|-2$ where the second inequality again follows from Lemma 1 . Since $1 \notin S_{\pi} \cup S$, the inequality $\left|S_{\pi}\right|+|S|>2 p_{1}(v)$ implies $p_{1}(v)=\frac{|N|-2}{2}$. Notice then that $\left|S_{\pi}\right|>|S| \geq p_{1}(v)+1=\frac{|N|}{2}$ holds with the first inequality following from $\pi \in \mathcal{P}^{*}$ and the second from $|S|>p_{1}(v)$. We have then $\left|S_{\pi}\right|+|S|>|N|$, which leads to a contradiction.

Proof of Proposition 6 We consider the two assertions separately.

(1) $\left|S_{\pi}\right| \geq p_{1}(v)+|\pi(1)| \operatorname{implies}\left(S_{\pi} ; \pi\right) \in E_{W}(v)$. 
Suppose that $\left|S_{\pi}\right| \geq p_{1}(v)+|\pi(1)|$. Let $S_{\pi}^{\prime} \subseteq S_{\pi}$ contain exactly $p_{1}(v)+1$ players. Consider the partition $\pi^{\prime}=\left\{\{1\}, S_{\pi}^{\prime}, \pi_{N \backslash\left(\pi(1) \cup S_{\pi}\right)}\right\} \cup\{\{r\}\}_{r \in(\pi(1) \backslash\{1\}) \cup\left(S_{\pi} \backslash S_{\pi}^{\prime}\right)}$. Notice that $\left(\pi^{\prime}(1) ; \pi^{\prime}\right)=\left(\{1\} ; \pi^{\prime}\right) \in E_{w}(v)$ would imply, by decisiveness of $v$, that $S_{\pi}^{\prime}$ loses against $\{1\}$ in $\pi^{\prime}$. By $\left|S_{\pi}^{\prime}\right|=p_{1}(v)+1>p_{1}(v)$, we have a contradiction to the definition of $p_{1}(v)$. So, $\left(\{1\} ; \pi^{\prime}\right) \notin E_{W}(v)$. By Lemma 2, each coalition in $\pi_{N \backslash\left(\pi(1) \cup S_{\pi}\right)}$ is of size at most $p_{1}(v)$, implying that $S_{\pi}^{\prime}$ is the unique largest coalition in $\pi^{\prime}$. We then have, by Proposition 5, that $\left(S_{\pi}^{\prime}, \pi^{\prime}\right) \in E_{W}(v)$.

Let $S_{\pi}^{\prime \prime}$ contain $S_{\pi}^{\prime}$ and $|\pi(1)|-1$ other members of $S_{\pi}$. Having in mind that $\left|S_{\pi} \backslash S_{\pi}^{\prime}\right|=\left|S_{\pi}\right|-p_{1}(v)-1 \geq|\pi(1)|-1$ is satisfied, it follows from applying growsymmetry $(|\pi(1)|-1)$-times and monotonicity of $v$ that $S_{\pi}^{\prime \prime}$ is winning in the partition $\pi^{\prime \prime}=\left\{S_{\pi}^{\prime \prime}, \pi(1), \pi_{N \backslash\left(\pi(1) \cup S_{\pi}\right)}\right\} \cup\{\{r\}\}_{r \in S_{\pi} \backslash S_{\pi}^{\prime \prime}}$. Further, by monotonicity of $v$, $\left(S_{\pi} ; \pi\right) \in E_{W}(v)$, showing that assertion (1) holds.

$$
\left|S_{\pi}\right|<p_{1}(v)+|\pi(1)| \operatorname{implies}(\pi(1) ; \pi) \in E_{W}(v) .
$$

Suppose that $\left|S_{\pi}\right|<p_{1}(v)+|\pi(1)|$. We split the proof in showing that $(\pi(1) ; \pi) \in E_{W}(v)$ follows when either $\left|S_{\pi}\right| \leq p_{1}(v)$ or $p_{1}(v)<\left|S_{\pi}\right|<p_{1}(v)+|\pi(1)|$ holds.

(2.1) $\left(\left|S_{\pi}\right| \leq p_{1}(v)\right.$ implies $\left.(\pi(1) ; \pi) \in E_{W}(v)\right)$. Suppose that $\left|S_{\pi}\right| \leq p_{1}(v)$. In view of Proposition 5, it is sufficient to show that $\left(S_{\pi} ; \pi\right) \in E_{W}(v)$ leads to a contradiction. For this, notice that, by the monotonicity of $v, S_{\pi}$ would be winning in the partition $\pi^{*}=\left\{S_{\pi}\right\} \cup\{\{i\}\}_{i \in N \backslash S_{\pi}}$. Let $\pi^{\prime}$ be a partition with respect to which $p_{v}(1)$ was calculated, that is, there exists $T \in \pi^{\prime}$ with $|T|=p_{v}(1)$ and $\left(\{1\} ; \pi^{\prime}\right) \in E_{W}(v)$. Take $S \subseteq T$ with $|S|=\left|S_{\pi}\right|$ (such a coalition $S$ does exist due to $|T|=p_{v}(1) \geq\left|S_{\pi}\right|$ ), and let $\pi^{\prime \prime}=\{S\} \cup\{\{i\}\}_{i \in N \backslash S}$ be the partition containing $S$ with all other players being single. By monotonicity of $v,\left(\{1\} ; \pi^{\prime \prime}\right) \in E_{W}(v)$ since $\{1\}$ is winning in $\pi^{\prime}$. Due to $1 \notin T \supseteq S$ and $1 \notin S_{\pi}$, the players in $S_{\pi} \cup S$ are switch-symmetric in $v$, and we can exchange the players from $S_{\pi}$ by those from $S$ (in the partition $\pi^{*}$ ) as to conclude that $S$ should be the winning coalition in $\pi^{\prime \prime}$. This gives a contradiction to $\left(\{1\} ; \pi^{\prime \prime}\right) \in E_{W}(v)$ and the decisiveness of $v$. Therefore, $\left(S_{\pi} ; \pi\right) \notin E_{W}(v)$, and by Proposition $5,(\pi(1) ; \pi) \in E_{W}(v)$.

(2.2) $\left(p_{1}(v)<\left|S_{\pi}\right|<p_{1}(v)+|\pi(1)|\right.$ implies $\left.(\pi(1), \pi) \in E_{W}(v)\right)$. Suppose that $p_{1}(v)<\left|S_{\pi}\right|<p_{1}(v)+|\pi(1)|$. Let $S_{\pi}^{\prime} \subset S_{\pi}$ contain exactly $p_{1}(v)$ players. Consider the partition $\pi^{\prime}=\left\{\{1\}, S_{\pi}^{\prime}, \pi_{N \backslash\left(\pi(1) \cup S_{\pi}\right)}\right\} \cup\{\{r\}\}_{r \in(\pi(1) \backslash\{1\}) \cup\left(S_{\pi} \backslash S_{\pi}^{\prime}\right)}$, and notice that, by Lemma 2, each coalition in $\pi_{N \backslash\left(\pi(1) \cup S_{\pi}\right)}$ is of size at most $p_{1}(v)$. The latter fact implies that the winning coalition in $\pi^{\prime}$ is either $\{1\}$ (if there is a coalition in $\pi_{N \backslash\left(\pi(1) \cup S_{\pi}\right)}$ of size $p_{1}(v)$ and by Corollary 1) or $S_{\pi}^{\prime}$ (if the size of each coalition in $\pi_{N \backslash\left(\pi(1) \cup S_{\pi}\right)}$ is less than $p_{1}(v)$ and by Proposition 5). Let us now show that $\left(S_{\pi}^{\prime} ; \pi^{\prime}\right) \in E_{W}(v)$ leads to a contradiction and thus, $\left(\{1\} ; \pi^{\prime}\right) \in E_{W}(v)$ should follow.

To get to a contradiction, suppose that $\left(S_{\pi}^{\prime} ; \pi^{\prime}\right) \in E_{W}(v)$. Consider the partition $\pi^{\prime \prime}=\left\{\{1\}, S_{\pi}^{\prime}\right\} \cup\{\{r\}\}_{r \in N \backslash\left(\{1\} \cup S_{\pi}^{\prime}\right)}$ and notice that, by the monotonicity of $v$, $\left(S_{\pi}^{\prime} ; \pi^{\prime \prime}\right) \in E_{W}(v)$. Let $\pi^{\prime \prime \prime}$ be a partition with respect to which $p_{v}(1)$ was calculated, that is, there exists $T \in \pi^{\prime \prime \prime}$ with $|T|=p_{v}(1)$ and $\left(\{1\} ; \pi^{\prime \prime \prime}\right) \in E_{W}(v)$. By monotonicity of $v,\left(\{1\} ; \pi^{i v}\right) \in E_{W}(v)$, where $\pi^{i v}$ is the partition containing $T$ with everyone 
else being single. Since $\left|S_{\pi}^{\prime}\right|=|T|=p_{v}(1)$, and all players in $S_{\pi}^{\prime} \cup T$ are switchsymmetric in $v$, in the partition $\pi^{\prime \prime}$, we can replace all players from $S_{\pi}^{\prime}$ by those from $T$ as to conclude that $T$ should be winning in the partition containing it with everyone else being single. Thus, we have a contradiction to $\left(\{1\} ; \pi^{i v}\right) \in E_{W}(v)$ and hence $\left(S_{\pi}^{\prime} ; \pi^{\prime}\right) \notin E_{W}(v)$, implying that $\left(\{1\} ; \pi^{\prime}\right) \in E_{W}(v)$.

Having in mind that $\left|S_{\pi}\right|<p_{1}(v)+|\pi(1)|$ implies $\left|S_{\pi}\right| \leq p_{1}(v)+|\pi(1)|-1$ and thus $|\pi(1)|-1 \geq\left|S_{\pi}\right|-p_{1}(v)=\left|S_{\pi} \backslash S_{\pi}^{\prime}\right|>0$, it follows from monotonicity and applying grow-symmetry $\left|S_{\pi} \backslash S_{\pi}^{\prime}\right|$-times that $Q^{*}$ is winning in the partition $\pi^{*}=\left\{Q^{*}, S_{\pi}, \pi_{N \backslash\left(\pi(1) \cup S_{\pi}\right)}\right\} \cup\{\{r\}\}_{r \in \pi(1) \backslash Q^{*}}$, where $Q^{*}$ contains player 1 and $\left|S_{\pi} \backslash S_{\pi}^{\prime}\right|$ other members of $\pi(1)$. By monotonicity of $v,(\pi(1) ; \pi) \in E_{W}(v)$.

Proof of Theorem 3 Let $v$ be a decisive and $(n-1)$-symmetric plurality game defined on the player set $N$, and let $v\left(\{1\} ; \pi^{a}\right)=1$. Take the weight vector $w \in X_{+}^{N}$ defined by $w_{1}=\frac{p_{1}(v)}{p_{1}(v)+|N|-1}$ and $w_{i}=\frac{1}{p_{1}(v)+|N|-1}$ for each $i \in N \backslash\{1\}$. In order to show that $w$ is supporting the game $v$, we explicitly consider the two cases in which $N$ contains either at most four players (Case A) ${ }^{5}$ or at least five players (Case B).

Case $A(|N| \leq 4)$ : For $|N|=2$, take $N=\{1,2\}$ and notice that $v$ is then defined by $v(\{1\} ;\{\{1\},\{2\}\})=1$ and $v(\{1,2\} ;\{\{1,2\}\})=1$. We have $p_{1}(v)=1$ and, by using the above construction of the weight vector, $w=\left(\frac{1}{2}, \frac{1}{2}\right)$. Clearly, the weight vector $w$ supports the game $v$.

If $|N|=3$, take $N=\{1,2,3\}$. Recall that $\{1\}$ is winning in the atomistic partition and, due to monotonicity of $v, \pi(1)$ is winning in all partitions $\pi \in \mathcal{P}$ with $|\pi(1)| \geq 2$. If $\{1\}$ additionally wins also in the partition $\{\{1\},\{2,3\}\}$, then $p_{1}(v)=2$ and we get $w=\left(\frac{1}{2}, \frac{1}{4}, \frac{1}{4}\right)$. In case that $\{2,3\}$ wins in $\{\{1\},\{2,3\}\}$, then $p_{1}(v)=1$ and $w=\left(\frac{1}{3}, \frac{1}{3}, \frac{1}{3}\right)$. It can be checked that, in either case, the constructed weights support the corresponding game.

Suppose finally that $|N|=4$, and let us take $N=\{1, j, k, \ell\}$. By assumption, $\{1\}$ is winning in the atomistic partition. Further, by monotonicity of $v$ and $(n-1)$ (grow-)symmetry, $\pi(1)$ is winning in all partitions $\pi \in \mathcal{P}$ with $|\pi(1)| \geq 2$. Consider now partitions of the type $\{\{1\},\{j, k\},\{\ell\}\}$ and notice that, by decisiveness, monotonicity, and $\{1\}$ being wining in the atomistic partition, it is impossible that $\{\ell\}$ wins in $\{\{1\},\{j, k\},\{\ell\}\}$. By $(n-1)$-(switch-)symmetry, if $\{j, k\}$ wins in that partition, it is also winning in all partitions of the same type. In such a case, monotonicity of $v$ requires $\{j, k, \ell\}$ to win in $\{\{1\},\{j, k, \ell\}\}$. We get then $p_{1}(v)=1$ and $w=\left(\frac{1}{4}, \frac{1}{4}, \frac{1}{4}, \frac{1}{4}\right)$. If it is player 1 who wins in all partitions of type $\{\{1\},\{j, k\},\{\ell\}\}$, then we get $p_{1}(v)=3$ (if $\{1\}$ wins in $\{\{1\},\{j, k, \ell\}\}$ ) or $p_{1}(v)=2$ (if $\{j, k, \ell\}$ wins in $\{\{1\},\{j, k, \ell\}\})$. The corresponding weight vectors supporting the games are then $\left(\frac{3}{6}, \frac{1}{6}, \frac{1}{6}, \frac{1}{6}\right)$ and $\left(\frac{2}{5}, \frac{1}{5}, \frac{1}{5}, \frac{1}{5}\right)$.

\footnotetext{
5 Recall that, by Theorem 1, every decisive plurality game with at most four players is weighted. What we show in Case $\mathrm{A}$ is that every decisive and $(n-1)$-symmetric plurality game with at most four players is supported by the weight vector $w$ specified above.
} 
Case $B(|N| \geq 5)$ : Take $\pi \in \mathcal{P}$ and notice that in view of Proposition 5 and by the fact that each player in $N \backslash\{1\}$ has the same weight according to $w$, it suffices to compare the weights of the coalitions $\pi(1)$ and a largest coalition in $\pi \backslash\{\pi(1)\}$. There are only two possible cases for the partition $\pi$ : either $\pi \in \mathcal{P}^{*}$ or $\pi \in \mathcal{P} \backslash \mathcal{P}^{*}$.

Case B.l $\left(\pi \in \mathcal{P}^{*}\right)$ : If $p_{1}(v)+|\pi(1)|>\left|S_{\pi}\right|$, then $(\pi(1) ; \pi) \in E_{W}(v)$ follows from Proposition 6. We have then

$$
w(\pi(1))=\frac{p_{1}(v)+|\pi(1)|-1}{p_{1}(v)+|N|-1} \geq \frac{\left|S_{\pi}\right|}{p_{1}(v)+|N|-1}=w\left(S_{\pi}\right) .
$$

On the other hand, if $\left|S_{\pi}\right| \geq p_{1}(v)+|\pi(1)|$, then we have by Proposition 6 that $\left(S_{\pi} ; \pi\right) \in E_{W}(v)$ and thus,

$$
w\left(S_{\pi}\right)=\frac{\left|S_{\pi}\right|}{p_{1}(v)+|N|-1}>\frac{p_{1}(v)+|\pi(1)|-1}{p_{1}(v)+|N|-1}=w(\pi(1)) .
$$

In either case, the game $v$ is supported by weight vector $w$.

Case B.2 $\left(\pi \in \mathcal{P} \backslash \mathcal{P}^{*}\right)$ : By Corollary $1,(\pi(1) ; \pi) \in E_{W}(v)$. Let $S$ be a largest coalition in $\pi \backslash\{\pi(1)\}$ and consider the following possibilities.

(B2.1) $\left(|N|\right.$ and $|N \backslash \pi(1)|$ are even numbers): We have in this case $|S| \leq \frac{|N|-|\pi(1)|}{2}$ and thus, $w(S) \leq \frac{|N|-|\pi(1)|}{2\left(p_{1}(v)+|N|-1\right)}$. On the other hand,

$$
\begin{aligned}
w(\pi(1)) & =\frac{p_{1}(v)+|\pi(1)|-1}{p_{1}(v)+|N|-1} \geq \frac{\frac{|N|-2}{2}+|\pi(1)|-1}{p_{1}(v)+|N|-1} \\
& =\frac{|N|-4+2|\pi(1)|}{2\left(p_{1}(v)+|N|-1\right)},
\end{aligned}
$$

where the inequality follows due to Lemma 1 . Notice further that $|N|-|\pi(1)|>$ $|N|-4+2|\pi(1)|$ would be fulfilled only if $|\pi(1)|=1$, which is in contradiction to both $|N|$ and $|N \backslash \pi(1)|$ being even numbers. We conclude that $|N|-4+2|\pi(1)| \geq|N|-|\pi(1)|$, and thus, $w(\pi(1)) \geq w(S)$.

(B2.2) $(|N|$ and $|N \backslash \pi(1)|$ are odd numbers): We have in this case $|\pi(1)| \geq 2$ and $|S| \leq \frac{|N|-|\pi(1)|-1}{2}$, and thus, $w(S) \leq \frac{|N|-|\pi(1)|-1}{2\left(p_{1}(v)+|N|-1\right)}$. On the other hand,

$$
\begin{aligned}
w(\pi(1)) & =\frac{p_{1}(v)+|\pi(1)|-1}{p_{1}(v)+|N|-1} \geq \frac{\frac{|N|-1}{2}+|\pi(1)|-1}{p_{1}(v)+|N|-1} \\
& =\frac{|N|-3+2|\pi(1)|}{2\left(p_{1}(v)+|N|-1\right)},
\end{aligned}
$$

where the inequality follows due to Lemma 1. In this case, $|N|-3+2|\pi(1)|>|N|-|\pi(1)|-1$ always holds and thus, $w(\pi(1))>w(S)$.

(B2.3) $\left(|N|\right.$ is even and $|N \backslash \pi(1)|$ is odd): We have in this case $|S| \leq \frac{|N|-|\pi(1)|-1}{2}$ and thus, $w(S) \leq \frac{|N|-|\pi(1)|-1}{2\left(p_{1}(v)+|N|-1\right)}$. On the other hand, 


$$
\begin{aligned}
w(\pi(1)) & =\frac{p_{1}(v)+|\pi(1)|-1}{p_{1}(v)+|N|-1} \geq \frac{\frac{|N|-2}{2}+|\pi(1)|-1}{p_{1}(v)+|N|-1} \\
& =\frac{|N|-4+2|\pi(1)|}{2\left(p_{1}(v)+|N|-1\right)},
\end{aligned}
$$

where the inequality holds due to Lemma 1. From $|N|-4+2|\pi(1)| \geq|N|-|\pi(1)|-1$, we conclude that $w(\pi(1)) \geq w(S)$.

(B2.4) $\left(|N|\right.$ is odd and $|N \backslash \pi(1)|$ is even): We have in this case $|S| \leq \frac{|N|-|\pi(1)|}{2}$ and thus, $w(S) \leq \frac{|N|-|\pi(1)|}{2\left(p_{1}(v)+|N|-1\right)}$. On the other hand,

$$
\begin{aligned}
w(\pi(1)) & =\frac{p_{1}(v)+|\pi(1)|-1}{p_{1}(v)+|N|-1} \geq \frac{\frac{|N|-1}{2}+|\pi(1)|-1}{p_{1}(v)+|N|-1} \\
& =\frac{|N|-3+2|\pi(1)|}{2\left(p_{1}(v)+|N|-1\right)},
\end{aligned}
$$

where the inequality holds due to Lemma 1. In this case, $|N|-3+2|\pi(1)| \geq|N|-|\pi(1)|$ holds, and thus, $w(\pi(1)) \geq w(S)$.

Proof of Proposition 7 Consider an externality-free decisive plurality game $v$ and suppose that, w.l.o.g., player 1 is the unique winner in the atomistic partition. Since there are no externalities, we have that $\{1\}$ is the unique winning coalition in any partition containing $\{1\}$. But then, by monotonicity of $v, \pi(1)$ is winning in every partition $\pi \in \mathcal{P}$. Taking the vector $w$ with $w_{1}=1$ and $w_{i}=0$ for each $i \in N \backslash\{1\}$ then shows that $v$ is weighted.

\section{References}

Albizuri MJ, Arin J, Rubio J (2005) An axiom system for a value partition function form. Int Game Theory Rev 7:63-73

Alonso-Meijide JM, Alvarez Mozos M, Fiestras-Janeiro M (2017) Power indices and minimal winning coalitions for simple games in partition function form. Group Decis Negot 26:1231-1245

Alvarez Mozos M, Alonso-Meijide JM, Fiestras-Janeiro M (2017) On the externality-free ShapleyShubik index. Games Econ Behav 105:148-154

Bolger EM (1986) Power indices for multicandidate voting games. Int J Game Theory 15(3):175-186

Carreras F, Freixas J (1996) Complete simple games. Math Soc Sci 32(2):139-155

de Clippel G, Serrano R (2008) Marginal contributions and externalities in the value. Econometrica 76:1413-1436

Dutta B, Ehlers L, Kar A (2010) Externalities, potential, value and consistency. J Econ Theory 145:2380-2411

Einy E, Lehrer E (1989) Regular simple games. Int J Game Theory 18:195-207

Elgot CC (1961) Truth functions realizable by single threshold organs. In: Switching circuit theory and logical design, AIEE conference paper 60-1311 (Oct. 1960), revised Nov. 1960, pp 341-345

Freixas J, Molinero X (2009) Simple games and weighted games: a theoretical and computational viewpoint. Discrete Appl Math 157:1496-1508

Gilboa I, Lehrer E (1991) Global games. Int J Game Theory 20(2):129-147 
Grabisch M, Funaki Y (2012) A coalition formation value for games in partition function form. Eur J Oper Res 221(1):175-185

Gvozdeva T, Slinko A (2011) Weighted and roughly weighted simple games. Math Soc Sci 61(1):20-30

Hafalir IE (2007) Efficiency in coalition games with externalities. Games Econ Behav 61(2):242-258

Hammer PL, Ibaraki T, Peled UN (1981) Threshold numbers and threshold completions. Ann Discret Math 11:125-145

Isbell JR (1958) A class of simple games. Duke Math J 25(3):423-439

Isbell JR (1956) A class of majority games. Q J Math 7(1):183-187

Koczy LA (2018) Partition function form games. Coalitional games with externalities, vol 48. Springer, Berlin

Lucas WF, Marcelli JC (1978) Discrete partition function games. In: Ordeshook PC (ed) Game theory and political science. New York University Press, New York, pp 191-213

Macho-Stadler I, Perez-Castrillo D, Wettstein D (2007) Sharing the surplus: an extension of the Shapley value for environments with externalities. J Econ Theory 135:339-356

Maskin E (2003) Bargaining, coalitions and externalities. President Address Econ Soc

McQuillin B (2009) The extended and generalized Shapley value. Simultaneous consideration of coalitional externalities and coalition structure. J Econ Theory 144:696-721

Myerson RB (1977) Values of games in partition function form. Int J Game Theory 6:23-31

Navarro N (2007) Fair allocation in networks with externalities. Games Econ Behav 58(2):354-364

Peleg B (1968) On weights of constant-sum majority games. SIAM J Appl Math 16:527-532

Ray D (2007) A game-theoretic perspective on coalition formation. Oxford University Press, Oxford

Shapley LS (1962) Simple games: an outline of the descriptive theory. Behav Sci 7:59-66

Sudhölter P (1996) The modified nucleolus as canonical representation of weighted majority games. Math Oper Res 21:734-756

Taylor AD, Zwicker WS (1999) simple games: desirability relations, trading, and pseudoweightings. Princeton University Press, Princeton

Taylor AD, Zwicker WS (1995) Simple games and magic squares. J Combi Theory Ser A 71:67-88

Taylor AD, Zwicker WS (1992) A characterization of weighted voting. Proc Am Math Soc 115(4):1089-1094

Thrall RM (1962) Generalised characteristic functions for n-person games. In: Proceedings of the Princeton University conference, pp 157-160

Thrall RM, Lucas WF (1963) N-person games in partition function form. Naval Res Logist Q 10(1):281-298

von Neumann J, Morgenstern O (1944) Theory of games and economic behavior. Princeton University Press, Princeton

Publisher's Note Springer Nature remains neutral with regard to jurisdictional claims in published maps and institutional affiliations. 